\title{
Estilos de socialización parental y adaptación de conducta en estudiantes del nivel secundario de una institución educativa de Lima Este, 2016
}

\author{
Styles of parental socialization and behavioral adaptation in secondary school students of an \\ educational institution in Lima Este, 2016
}

Janeth Rosita Pérez Díaz ${ }^{*}$, Yesennia Páucar Alcalá², Daniel Farfán Rodríguez³

\begin{abstract}
RESUMEN
Objetivo: Determinar la relación entre estilos de socialización parental y adaptación de conducta. Material y métodos: El estudio responde a un diseño no experimental, de alcance descriptivo correlacional y corte transversal. La muestra estuvo conformada por 200 alumnos del cuarto y quinto año del nivel secundario de una institución educativa de Lima Este. Los instrumentos utilizados fueron el Inventario de Adaptación de Conducta IAC (De la Cruz y Cordero, 1990) adaptado por Ruiz (1995) y la Escala de Estilos de Socialización Parental en la Adolescencia ESPA 29 de Musitu y García (2004) adaptada por Jara (2013). Resultados: Los resultados indican que no existe asociación entre estilos de socialización de las madres y adaptación de conducta en adolescentes (chi2= 4,320; p= ,633), tampoco se encontró relación entre estilos de socialización del padre y adaptación de conducta en adolescentes (chi2= 9,616; p= ,142). Sin embargo, la aceptación/implicación de la madre (rho= ,153; p= ,030), coerción/imposición del padre (rho= -,164; p= ,021), y aceptación/implicación del padre (rho= ,290; $\mathrm{p}=, 000$ ) obtuvieron relación significativa con la adaptación de conducta en adolescentes. Pero la coerción/ imposición de la madre (rho= -,109; p= ,123) no correlacionó con la adaptación conductual. Conclusión: Los resultados indican que no existe asociación entre estilos de socialización de las madres y adaptación de conducta en adolescentes.
\end{abstract}

Palabras clave: Estilos de socialización parental, adaptación conductual, adolescencia, aceptación/implicación, coerción/imposición.

\begin{abstract}
Objective: The aim of this research was to determine the relationship between styles of socialization and adaptation of conduct. Mtehods: The study responds to a non-experimental, correlational and cross-sectional descriptive scope. The sample consisted of 200 students in the fourth and fifth year secondary level educational institution of this Lima. The instruments used were the Inventory Adjustment of Conduct - IAC (De la Cruz \& Cordero, 1990) adapted by Ruiz (1995) and the Parental Styles Socialization Scale for Adolescence - ESPA 29 (Musitu \& Garcia, 2004) adapted by Jara (2013). Results: The results indicate that there is no association between the styles of socialization of mothers and adaptation of behavior in adolescents (chi2 $=4.320$; $\mathrm{p}=$, 633), no relationship between the styles of socialization of father and adaptation of behavior in adolescents was found (chi2 = 9,616, p =, 142). However, acceptance / involvement of mother (rho = 153; p =, 030), coercion / imposition of father (rho = -, 164; $\mathrm{p}=, 021$ ), and acceptance / involvement of parents (rho =, 290; $\mathrm{p}=.000$ ) had significant relationship with the adaptation of behavior in adolescents. But coercion / imposition of mother (rho $=-, 109 ; \mathrm{p}=, 123)$. Conclusions: did not correlate with behavioral adaptation.
\end{abstract}

Keywords: Styles of parental socialization, behavioral adaptation, adolescence, acceptance / implication, coercion / imposition.

\footnotetext{
${ }^{1}$ Bachiller en Psicología, Facultad de Ciencias de la Salud, Universidad Peruana Unión, Lima, Perú

${ }^{2}$ Bachiller en Psicología, Facultad de Ciencias de la Salud, Universidad Peruana Unión, Lima, Perú

${ }^{3}$ Docente de EP de Psicología, Facultad de Ciencias de la Salud, Universidad Peruana Unión, Lima, Perú
} 
Estilos de socialización parental y adaptación de conducta en estudiantes del nivel secundario de una institución educativa de Lima Este, 2016

\section{INTRODUCCIÓN}

En la actualidad los padres se incorporan a actividades laborales que requieren mayor tiempo fuera del hogar; por esta razón, se da el incumplimiento parcial o total del rol parental, impidiendo a los hijos adquirir habilidades que posibiliten un funcionamiento saludable en la vida social (Cárdenas, 2013).

La encuesta mundial de valores a nivel de 57 países, realizada entre los años 2005 al 2008, señala que el 90.1\% de los participantes consideran como muy importante a la familia en comparación con el trabajo, religión, amigos, tiempo libre y política, los que obtuvieron menores porcentajes (Pliego, 2013).

El Instituto Nacional Estadística e Informática (INEI, 2014) refiere que, en la interacción familiar, el 74,9\% de los padres y el 72,6\% de las madres presentan represión verbal, considerándolo como una de las principales formas de castigo.

Asimismo, la socialización parental influye en distintas variables como el juicio crítico, independencia y valores, necesarios para el desarrollo de la adaptación social en la etapa de la adolescencia (Blanco, 2007). En ese sentido, Alvarado y Cruz (2004) afirman que el ambiente familiar en el que se desarrolla un individuo ejerce influencia en los cambios adaptativos en su conducta. Por ello el hogar en el que se vivencian relaciones caracterizadas por el respeto, honradez, responsabilidad, obediencia, amor permite desarrollar control de impulsos y dominio propio. Asimismo, Cuervo (2010) enfatiza que los cambios en la estructura dinámica, de una familia, pueden tener un efecto en la educación y desarrollo socioafectivo de sus miembros, en especial de los hijos. Es así que, Fuentes et al. (2015) realizaron un estudio cuyos resultados indicaron una correlación altamente significativa y en sentido negativo entre el componente aceptación/implicación parental y algunos indicadores de desajuste psicológico, como hostilidad/agresión, autoestima negativa, autoeficacia negativa, irresponsividad emocional, entre otros. Diversas teorías tales como el modelo bidimensional de socialización propuesto por Musitu y García (2001) refieren que el primer eje de cohesión/imposición está relacionado con los estilos de paternidad autoritario y negligente, mientras que el segundo eje implicación/ aceptación está relacionado con los estilos de paternidad, autoritativo e indulgente. De manera que los padres con altos niveles de implicación/ aceptación muestran afecto y cariño a sus hijos cuando se comportan adecuadamente o no, dialogando y razonando con ellos; siendo diferente en los padres con bajos niveles de implicación/aceptación, mostrando indiferencia ante las conductas adecuadas de sus hijos, no conversan con ellos se muestran indiferentes ante sus conductas. Los padres con altos niveles en la dimensión coerción/ imposición, exigen a sus hijos que no vuelvan a realizar conductas que ellos no desean muy a pesar de razonar o no con ellos, la coacción puede ser física o verbal y puede consistir en privarle de las cosas que más le agradan. De la Cruz y Cordero (1990) sostuvieron que el ajuste comportamental y acepción a las normas establecidas en tres áreas a las que los estudiantes deben adaptarse: la escolar, la familiar y la personal, reflejarán las habilidades sociales, emocionales y cognitivas.

\section{MATERIALES Y MÉTODOS}

Del total de la muestra que corresponde a los estudiantes, se observa que el $52,5 \%$ es del sexo femenino el 62,0\% es entre 14 y 15 años de edad, el $69,5 \%$ procede de la costa y 52,5\% cursa el quinto año de secundaria.

Tabla 1

Características sociodemográficas de los participantes

\begin{tabular}{cccc}
\hline \multicolumn{2}{c}{ Datos sociodemográficos } & $\mathrm{n}$ & $\%$ \\
\hline \multirow{4}{*}{ Sexo } & Masculino & 95 & 47,5 \\
& Femenino & 105 & 52,5 \\
& De 14 a 15 & 124 & 62,0 \\
& años & & \\
& De 16 a 18 & 76 & 38,0 \\
Procedencia & años & & \\
& Costa & 139 & 69,5 \\
& Sierra & 40 & 20,0 \\
& Selva & 21 & 10,5 \\
Grado & Cuarto & 95 & 47,5 \\
& Quinto & 105 & 52,5 \\
\hline
\end{tabular}

\section{Instrumentos}

Para la investigación se empleó la Escala de Socialización Parental en la Adolescencia (ESPA 29, 2013) constituida por 29 ítems con una estructura de tipo escala Likert, con cuatro opciones de respuesta: nunca, algunas veces, muchas veces y siempre. Cabe señalar que la utilidad del instrumento es evaluar las relaciones entre padres e hijos mediante la valoración de las reacciones de los progenitores ante 29 situaciones relevantes de la vida diaria. Tiene una consistencia interna global de $(911)$ en la madre y (,926) del padre, respecto a la dimensión aceptación/ 
implicación de la madre es de ,941 y ,879 del padre, evidenciando que el instrumento es fiable y altamente confiable. A su vez se encontraron correlaciones entre las dimensiones y el constructo general: $(, 797)$ en afecto; (,701) en indiferencia (,706) en diálogo; (,632) en displicencia; (,765) en coerción verbal; (,509) en coerción física y (,822) en privación, siendo altamente significativos.

Por otro lado, se utilizó el inventario de adaptación de conducta (I.A.C, Cruz y Cordero, 1995). Es un instrumento de autoinforme que consta por 123 ítems diseñado para valorar el grado de adaptación de los adolescentes entre los 12 y 17 años de edad, de manera individual o colectiva. Para la evaluación se contó con un valor de confiabilidad del alfa de Crombach de .0.90, por lo que el instrumento es considerado válido y confiable. De acuerdo a las subdimensiones, hay una confiabilidad de 0.95 en el alfa de Crombach global, 0.92 en el área personal, 0.89 en el área familiar, 0.90 en el área educativa y 0.85 en el área social.

\section{Análisis estadístico}

Para el análisis estadístico se empleó el Software estadístico SPSS para Windows versión 22, aplicado a las ciencias sociales. Se inició con la creación de una base de datos con la codificación para cada elemento a ingresarse como variable. Posterior al ingreso de las respuestas de los participantes, se procedió con la limpieza de los datos. A continuación, se procedió a realizar los análisis descriptivos utilizando tablas de frecuencia y el análisis de normalidad, en base a cuyos resultados se optó por proceder a seleccionar una prueba no paramétrica para la correlación. Es por ello que el análisis de la correlación se realizó con el estadístico Rho de Spearman.

\section{RESULTADOS}

Para contrastarlas hipótesis planteadas, sehaprocedido a realizar primero la prueba de bondad de ajuste para precisar si las variables presentan una distribución normal. Se observa que los datos no presentan una distribución normal, dado que el coeficiente obtenido (K-S) no es significativo ( $\mathrm{p}>0.05$ ). Por tanto, para los análisis estadísticos correspondientes se empleará estadística no paramétrica. Asimismo, en la tabla 3 se muestran los puntajes de las dimensiones y las puntuaciones totales de prácticas de crianza parental y ansiedad.

Tabla 2

Prueba de Chi cuadrado para la asociación entre estilos de socialización de la madre y la adaptación conductual

\begin{tabular}{lccc}
\hline & \multicolumn{2}{c}{ Estilos de socialización de la madre } & \\
& Chi-cuadrado & $\mathrm{Gl}$ & $\mathrm{p}$ \\
\hline Adaptación general & $4,320 \mathrm{a}$ & 6 &, 633 \\
Adaptación personal & $3,969 \mathrm{~b}$ & 6 &, 681 \\
Adaptación familiar & $12,202 \mathrm{c}$ & 6 &, 058 \\
Adaptación escolar & $3,172 \mathrm{~d}$ & 6 &, 787 \\
Adaptación social & $7,799 \mathrm{e}$ & 6 &, 253 \\
\hline
\end{tabular}

a 7 casillas (58,3\%) han esperado un recuento menor que 5. El recuento mínimo esperado es ,22.

b. 6 casillas $(50,0 \%)$ han esperado un recuento menor que 5 . El recuento mínimo esperado es 1,06.

c. 7 casillas $(58,3 \%)$ han esperado un recuento menor que 5 . El recuento mínimo esperado es ,32.

d. 8 casillas $(66,7 \%)$ han esperado un recuento menor que 5 . El recuento mínimo esperado es, 04.

e. 6 casillas $(50,0 \%)$ han esperado un recuento menor que 5 . El recuento mínimo esperado es ,52.

En la Tabla 2 se presenta la asociación entre estilos de socialización percibida en la madre y la adaptación conductual de los adolescentes. Se aprecia que en la adaptación general el chi cuadrado es $(4,320)$ y el valor de p es (,633), por lo que no existe asociación significativa entre ambas variables. Para la adaptación personal, el chi cuadrado es $(3,969)$ y el valor de p es (,681), por lo que se concluye que no existe asociación significativa. Respecto a la adaptación familiar, el chi cuadrado es de $(12,202)$ y el valor de p es $(, 058)$ llegando a establecer que no existe asociación significativa. En la adaptación escolar, el chi cuadrado es $(3,172)$ y el valor de p es (,787); lo cual representa que no existe asociación significativa. Finalmente, en la adaptación social el chi cuadrado es de $(7,799)$ y el valor de p es $(, 253)$ asumiendo que no hay asociación significativa entre las variables. 
Estilos de socialización parental y adaptación de conducta en estudiantes del nivel secundario de una institución educativa de Lima Este, 2016

Tabla 3

Estilos de socialización del padre y adaptación de conducta

\begin{tabular}{lrcc}
\hline & \multicolumn{2}{c}{ Estilos de socialización del padre } & \\
& Chi-cuadrado & gl & P \\
\hline Adaptación general & $9,616 \mathrm{a}$ & 6 &, 191 \\
Adaptación personal & $8,701 \mathrm{~b}$ & 6 &, 000 \\
Adaptación familiar & $28,769 \mathrm{c}$ & 6 &, 420 \\
Adaptación escolar & $6,028 \mathrm{~d}$ & 6 &, 151 \\
Adaptación social & $9,416 \mathrm{e}$ & 6 & \\
\hline
\end{tabular}

a. 6 casillas $(50,0 \%)$ han esperado un recuento menor que 5 . El recuento mínimo esperado es ,39.

b. 6 casillas $(50,0 \%)$ han esperado un recuento menor que 5 . El recuento mínimo esperado es 1,86.

c. 7 casillas $(58,3 \%)$ han esperado un recuento menor que 5 . El recuento mínimo esperado es ,56.

d. 7 casillas $(58,3 \%)$ han esperado un recuento menor que 5 . El recuento mínimo esperado es 07.

e. 6 casillas (50,0\%) han esperado un recuento menor que 5. El recuento mínimo esperado es ,91.

En la Tabla 3 se presenta la asociación entre estilos de socialización percibida en el padre y la adaptación conductual de los adolescentes, con respecto a la adaptación general el chi cuadrado es 9,616 y el valor de p es ,142, por lo que se asume que no existe asociación significativa entre ambas variables. Para la adaptación personal, el chi cuadrado es 8,701 y el valor de p es, 191 , por lo que se concluye que no existe asociación significativa. En la adaptación familiar, el chi cuadrado es de 28,769 y el valor de $\mathrm{p}$ es ,000, llegando a establecer que no existe asociación significativa. Se aprecia en la adaptación escolar, el chi cuadrado es 6,028 y el valor de p es ,420; lo cual representa que no existe asociación significativa. Finalmente, en la adaptación social, el chi cuadrado es de 9,416 y el valor de p es ,151 asumiendo que no hay asociación significativa entre las variables.

Tabla 4

Correlación entre dimensiones de socialización parental y adaptación de conducta

\begin{tabular}{lcc}
\hline & \multicolumn{2}{l}{$\begin{array}{l}\text { Adaptación de } \\
\text { conducta general }\end{array}$} \\
\cline { 2 - 3 } & Rho & P \\
\hline Madre Aceptación/Implicación &, $153^{*}$ & 0,03 \\
Madre Coerción/Imposición & $-0,109$ & 0,123 \\
Padre Aceptación/Implicación &, $290^{* *}$ & 0 \\
Padre Coerción/Imposición &,$- 164^{*}$ & 0,021 \\
\hline
\end{tabular}

* La correlación es significativa en el nivel 0,05 (2 colas).

** La correlación es significativa en el nivel 0,01 ( 2 colas)

En la Tabla 4 se presenta la correlación entre aceptación/implicación de la madre y la adaptación conductual de los adolescentes, el coeficiente de Spearman muestra un grado de intensidad rho ,153 y el valor $\mathrm{p}=, 030$ muestra que existe relación significativa en sentido positivo entre ambas variables.
En la dimensión coerción/imposición de la madre y la adaptación conductual de los adolescentes, el coeficiente de Spearman muestra un grado de intensidad rho,- 109 y el valor $p=, 123$ muestra que no existe relación significativa en sentido negativo entre ambas variables.

En la dimensión aceptación/dimensión del padre y la adaptación conductual de los adolescentes, el coeficiente de Spearman muestra un grado de intensidad rho ,290 y el valor $\mathrm{p}=, 000$ muestra que existe relación significativa en sentido positiva entre ambas variables.

En la dimensión coerción/imposición del padre y la adaptación conductual de los adolescentes, el coeficiente de Spearman muestra un grado de intensidad rho,- 164 y el valor $\mathrm{p}=, 021$ muestra que existe relación altamente significativa en sentido negativo entre ambas variables.

\section{DISCUSIÓN}

Al realizar los análisis para determinar la relación entre estilos de socialización parental de la madre y el padre, y la adaptación de conducta en estudiantes del nivel secundario, se evidencia que no existe asociación entre las variables mencionadas. Esto significa que la experiencia de adecuación personal, académica, social y familiar se desarrolla independientemente de los patrones correctivos o reacciones que manifiestan los padres frente a las conductas positivas o negativas de los adolescentes. Este resultado contradice a Musitu y García (2004) quienes proponen que el estilo de socialización parental tiene un impacto en la relación paterna-filial, y el proceso por el que la persona desarrolla interacciones con otros individuos, en base a aspectos sociales y culturales (García, 
Magaz y García, 2011), y que, por lo tanto, tendría influencia en el modo en que los adolescentes se adaptan a su medio. Sin embargo, según los resultados de este estudio, los patrones de interacciones paternofiliales no tienen una influencia relevante en el ajuste conductual de los adolescentes. En opinión de Estévez, Jiménez y Musitu (2011) los estilos de socialización parental varían de acuerdo a la cultura donde se desarrolla la familia. En este sentido, se sustenta que los resultados obtenidos no coincidan con lo propuesto por la literatura revisada también por las variables mediadoras, como el entorno social que esté interviniendo, ya que la etapa de la adolescencia y sus interacciones se deben, en mayor medida, en el entorno social.

En cuanto a la relación entre aceptación-implicación de la madre y adaptación de conducta en estudiantes del nivel secundario, el resultado fue rho ,153*, ( $\mathrm{p}=$ ,030), lo que indica que existe relación significativa en sentido positivo. Por lo tanto, se entiende que cuando las madres demuestran afecto mediante halagos, consejos, cuidado y delegan responsabilidades, tomando en cuenta las opiniones de los hijos, aun cuando los hijos presentan conductas inadecuadas los madres dialogan con estos y le explican los efectos de su comportamiento negativo y las razones por qué debe cambiar estas conductas; posibilitando tener una mejor adaptación en su medio social, familiar, escolar, pues les permiten tomar sus propias decisiones en un clima de seguridad emocional. En el mismo sentido, Cabrera, Guevara y Barrera (2006) refieren que los padres que dedican mayor tiempo a la convivencia armoniosa con sus hijos crean un clima de protección y cuidado que aumentan las conductas adaptativas de los menores; asimismo, disminuye la violencia y rebeldía. Por el contrario, cuando hay ausencia de normas, no se logran adecuadas relaciones interpersonales y los hijos desarrollan comportamientos ansiosos y depresivos. De la Cruz y Cordero (1990) refieren que el ajuste comportamental a las normas establecidas en el hogar permite adaptarse al medio escolar, familiar y personal reflejando habilidades sociales, emocionales y cognitivas.

En cuanto a la relación entre coerción-imposición de la madre y adaptación de conducta en estudiantes del nivel secundario, se muestra el grado de intensidad de rho, -,109 ( $\mathrm{p}=$,123) indicando que no existe relación significativa. Esto quiere decir que, aunque la madre utilice amenazas verbales como regaños, reproches y privación de algún objeto, no tiene un impacto significativo en la forma cómo los hijos se adaptan en su medio personal, académico, social y familiar. Estas prácticas correctivas de la madre son, en su mayoría, de tipo verbal y de privación que, a la vez, no son efectivas para lograr el ajuste en los hijos. A diferencia de lo que se observa en la coerción ejercida por los padres, quienes utilizan con más frecuencia el castigo físico y menos castigo verbal y privación, logrando generar cambios en la adaptación de los hijos.

En cuanto a la relación entre aceptación-implicación del padre y adaptación de conducta en estudiantes del nivel secundario, se muestra el grado de intensidad de rho, $290 * *(p=, 000)$ indicando que existe relación altamente significativa en sentido positivo. Es decir, los padres que demuestran cariño mediante palabras de reconocimiento y dialogan con sus hijos para hacerlos razonar sobre su mala conducta, les posibilitan un mejor desenvolvimiento en su ámbito social y adaptación a su ambiente tanto personal, social como escolar; ya que, si hay una adecuada comunicación con los hijos, y buenas relaciones, ellos serán capaces de interiorizar los valores y normas sociales, llegando a ser personas autónomas y responsables (Martínez y García, 2007). Asimismo, es importante que los padres tengan una relación afectiva y comunicativa adecuada con sus hijos, repercutiendo en el comportamiento de los hijos, así cuando se presente una conducta inadecuada en los hijos, los padres podrán dialogar con ellos para cambiar la conducta negativa (Musitu y Garcia, 2004).

En cuanto a la relación entre coerción-imposición del padre y adaptación de conducta en estudiantes del nivel secundario, se muestra el grado de intensidad de rho, -,164*, $(\mathrm{p}=$,021) indicando que sí existe relación significativa en sentido negativo. Esto quiere decir que los padres quienes ejercen castigos físicos y verbales ante conductas inadecuadas influyen negativamente en sus hijos adolescentes, provocando resentimientos y conflictos, afectando el ajuste familiar. Kagan y Moss (1965) explican que cuando los padres controlan el comportamiento de sus hijos imponiendo muchos límites, obteniendo una obediencia estricta apoyándose en castigos físicos, privaciones y amenaza verbal; pueden adquirir ajuste a las reglas y normas de un modo superficial, pero en el futuro generan rebeldía y falta de motivación (Educando, 2012). Asimismo, la restricción, la reprensión y la exigencia que los padres tienen con sus hijos, para cumplir algo determinado, influye de manera negativa en el comportamiento de estos (Musitu y García 2004).

\section{Declaración de financiamiento y de conflicto de intereses:}

El estudio fue financiado por los autores, quienes declaran no tener algún tipo de conflicto de interés en la investigación realizada. 
Estilos de socialización parental y adaptación de conducta en estudiantes del nivel secundario de una institución educativa de Lima Este, 2016

\section{Correspondencia:}

Janeth Rosita Pérez Díaz

Facultad de Ciencias de la Salud, Universidad
Peruana Unión, Carretera central Km 19, Ñaña, Perú. Tel.: 944576683

e-mail: rositajanthperezdiaz@hotmail.com

\section{REFERENCIAS BIBLIOGRÁFICAS}

Alvarado, A. y Cruz, A. (2004). La conducta antisocial en adolescentes, su relación con el entorno familiar y la percepción de riesgo social, una base para la prevención de conductas delictivas. (Tesis de licenciatura, Universidad Autónoma de México). Recuperado de http://www.uade.inpsiquiatria.edu.mx/ pagina_contenidos/tesis/tesis_aurora.pdf

Cárdenas, K. (2013). Asociación entre estilos de socialización parental y habilidades sociales del adolescente en una institución educativa nacionalV.M.T. (Tesis de licenciatura, Universidad Ricardo Palma). Lima. Perú. Recuperado de http://cybertesis. urp.edu.pe/bitstream/urp/309/1/Cardenas_kp.pdf

Cuervo, A. (2010). Pautas de crianza y desarrollo socioafectivo en la infancia. Diversitas, 6(1), 111-121. Recuperado de http://revistas.usantotomas.edu.co/idex. php/diversitas/article/view/163

De la Cruz, M. y Cordero, A. (1990). Inventario de adaptación de conducta. Madrid: TEA.

Educando (2012). Familia y escuela en la adaptación escolar del niño. Recuperado de http://www.educando. edu.do/articulos/familia/familia-y-escuela-en-laadaptacion-escolar-del-nino/

Estévez, E., Jiménez, T. y Musitu, G. (2011). Relación entre padres e hijos adolescentes. España: Cultural Valencianas. Recuperado de http://books.com.pe/ books?id=T9xaFGC6VZ8C\&\%dq=socializaci\%C3\% B3n+parental\&hl=es\&source=gbs_navlinks_s

García, E., Magaz, A. y García, R. (2011). Escala Magallanes de Adaptación. España: Bizkaia.
Recuperado de http: //www.gac.com.es/editorial/INFO/ Manuales/ema MANU.pdf

Instituto Nacional Estadística e Informática (INEI, 2014). Perú: Encuesta demográfica $y$ de salud familiar, 79. Recuperado de http://www.inei.gob.pe/media/ MenuRecursivo/publicacionesdigitales/Est/Lib1211/ idex.html

Kagan, J. y Moss, H. (1965). Bith to maturity. New York: wiley. 11(1), 77-88. Recuperado de: http://www.jstor. org/stable/23082501.

Martinez, I. y García, J. (2007). Impact of parenting styles on adolescents self esteem and internalization of values in spain. Spanish journal of psycology, 10(2), 458_467. Recuperado de: www.ncbi.nlm.nih.gov/ pubmed/17992960.

Musitu, G., y García, F. (2001). Escala de estilos de socialización parental en la adolescencia. Madrid: TEA.

Musitu, G., y García, F. (2004). Consecuencia de la socialización familiar en la cultura española. Psicothema, 16(2), 297-302. Recuperado de http:// www.psicothema.com/pdf/1196.pdf

Yubero, S., Larrañaga, E. y Blanco. A. (2007). Convivir con la violencia: un análisis desde la psicología y la educación de la violencia en nuestra sociedad. España: Universidad de Castilla-La Mancha

Recibido: 13/07/2016 Aceptado: 05/09/2016 